\title{
Generating functions of binary products of k-Fibonacci and orthogonal polynomials
}

\author{
Ali Boussayoud ${ }^{1} \cdot$ Souhila Boughaba ${ }^{1} \cdot$ Mohamed Kerada $^{1} \cdot$ Serkan $^{\text {Araci }}{ }^{2}$. \\ Mehmet Acikgoz ${ }^{3}$
}

Received: 22 September 2017 / Accepted: 28 January 2019 / Published online: 4 February 2019

(c) The Royal Academy of Sciences, Madrid 2019

\begin{abstract}
In this paper, we introduce a new operator in order to derive some new symmetric properties of $k$-Fibonacci and $k$-Pell numbers and Tchebychev polynomials of first and second kind. By making use of the new operator defined in this paper, we give some new generating functions for $k$-Fibonacci and $k$-Pell numbers and Fibonacci polynomials.
\end{abstract}

Keywords Symmetric functions $\cdot$ Generating functions $\cdot k$-Fibonacci numbers $\cdot k$-Pell numbers

Mathematics Subject Classification 05E05 · 11B39

\section{Introduction}

In mathematics, orthogonal polynomials consist of polynomials such that any two different polynomials in the sequence are orthogonal to each other under some inner product. The most

\footnotetext{
Ali Boussayoud

aboussayoud@yahoo.fr

Souhila Boughaba

souhilaboughaba@gmail.com

Mohamed Kerada

mkerada@yahoo.fr

Serkan Araci

mtsrkn@hotmail.com

Mehmet Acikgoz

acikgoz@gantep.edu.tr

1 LMAM Laboratory, Department of Mathematics, Mohamed Seddik Ben Yahia University, Jijel, Algeria

2 Department of Economics, Faculty of Economics, Administrative and Social Sciences, Hasan Kalyoncu University, 27410 Gaziantep, Turkey

3 Department of Mathematics, Faculty of Arts and Science, University of Gaziantep, 27310 Gaziantep, Turkey
} 
widely used orthogonal polynomials are the classical orthogonal polynomials (Tchebychev polynomials of first and second kinds, Fibonacci polynomials).

Further in [1], generating functions of the incomplete Fibonacci and Lucas numbers are determined. In [2], Djordjevic gave the incomplete generalized Fibonacci and Lucas numbers. In [3], Djordjevic and Srivastava defined incomplete generalized Jacobsthal and JacobsthalLucas numbers. In [4], the authors define the incomplete Fibonacci and Lucas numbers. Also the authors define the incomplete bivariate Fibonacci and Lucas $p$-polynomials in [5].

On the other hand, many kinds of generalizations of Fibonacci numbers have been presented in the literature. In particular, a generalization is the $k$-Fibonacci Numbers. For any positive real number $k$, the $k$-Fibonacci sequence, say $\left(F_{n, k}\right)_{n \in \mathbb{N}}$, is defined recurrently by

$$
\left\{\begin{array}{l}
F_{k, 0}=1, \quad F_{k, 1}=k \\
F_{k, n+1}=k F_{k, n}+F_{k, n-1}, \quad n \geq 1
\end{array}\right.
$$

The $k$-Pell numbers have been defined in [6] for any number $k$ as follows:

$$
\left\{\begin{array}{l}
P_{k, 0}=0, \quad P_{k, 1}=1 \\
P_{k, n+1}=2 P_{k, n}+k P_{k, n-1}, \quad n \geq 1 .
\end{array}\right.
$$

In this contribution, we shall define a new useful operator denoted by $\delta_{e_{1} e_{2}}^{-k}$ for which we can formulate, extend and prove new results based on our previous ones, see [7-9]. In order to determine generating functions for $k$-Fibonacci and $k$-Pell numbers and Tchebychev polynomials of the first and second kind and Fibonacci polynomials, we combine between our indicated past techniques and these presented polishing approaches.

In Sect. 2, we introduce a new symmetric function and give some properties of this symmetric function. We also give some more useful definitions which are used in the subsequent sections. In Sect. 3, we prove our main result which relates the symmetric function defined in the previous section with the symmetrizing operator. This main theorem unifies several previously known results about the generating functions. It is then used to find the product of $k$-Fibonacci numbers identities and the generating functions for the product of $k$-Fibonacci numbers and of $k$-Pell numbers, in Sect. 4. In Sect. 5 Generatring functions of some wellknown numbers and polynomials.

\section{Definitions and some properties}

In this section, we introduce a new symmetric function and give some properties of this symmetric function. We also give some more useful definitions from the literature which are used in the subsequent sections.

We shall handle functions on different sets of indeterminates (called alphabets, though we shall mostly use commutative indeterminates for the moment). A symmetric function of an alphabet $A$ is a function of the letters which is invariant under permutation of the letters of $A$. Taking an extra indeterminate $z$, one has two fundamental series

$$
\lambda_{z}(A)=\Pi_{a \in A}(1+z a), \sigma_{z}(A)=\frac{1}{\Pi_{a \in A}(1-z a)},
$$

the expansion of which gives the elementary symmetric functions $\Lambda_{n}(A)$ and the complete functions $S_{n}(A)$ :

$$
\lambda_{z}(A)=\sum_{n=0}^{\infty} \Lambda_{n}(A) z^{n}, \quad \sigma_{z}(A)=\sum_{n=0}^{\infty} S_{n}(A) z^{n} .
$$


Let us now start at the following definition.

Definition 1 Let $A$ and $B$ be any two alphabets, then we give $S_{n}(A-B)$ by the following form:

$$
\frac{\Pi_{b \in B}(1-z b)}{\Pi_{a \in A}(1-z a)}=\sum_{n=0}^{\infty} S_{n}(A-B) z^{n}=\sigma_{z}(A-B),
$$

with the condition $S_{n}(A-B)=0$ for $n<0$ (see [10]).

Corollary 1 Taking $A=0$ in (2.1) gives

$$
\Pi_{b \in B}(1-z b)=\sum_{n=0}^{\infty} S_{n}(-B) z^{n}=\lambda_{z}(-B) .
$$

Further, in the case $A=0$ or $B=0$, we have

$$
\sum_{n=0}^{\infty} S_{n}(A-B) z^{n}=\sigma_{z}(A) \times \lambda_{z}(-B)
$$

Thus,

$$
S_{n}(A-B)=\sum_{k=0}^{n} S_{n-k}(A) S_{k}(-B) \quad(\text { see }[11]) .
$$

Since the summation is, indeed, limited to a finite number of nonzero terms, we have

$$
S_{n}(x-B)=x^{n} S_{0}(-B)+x^{n-1} S_{1}(-B)+x^{n-2} S_{2}(-B)+\ldots,
$$

where $S_{k}(-B)$ are the coefficients of polynomials $S_{n}(x-B)$ for $0<k<n$.

Notice that $S_{k}(-B)=0$ for $k>n$. Let $B=\{b, b, \ldots, b\}$ be an alphabet of cardinality $n$, we have

$$
S_{n}(x-b-b-b-\cdots-b)=(x-b)^{n} \text {. (see [7]) }
$$

Choosing $B=\{1,1,1, \ldots, 1\}$ yields to two binomial coefficients as

$$
S_{k}(-n)=(-1)^{k}\left(\begin{array}{l}
n \\
k
\end{array}\right) \text { and } S_{k}(n)=\left(\begin{array}{c}
n+k-1 \\
k
\end{array}\right) \text {. }
$$

Definition 2 Let $g$ be any function on $\mathbb{R}^{n}$, then we consider the divided difference operator as the following form

$$
\partial_{x_{i} x_{i+1}}(g)=\frac{g\left(x_{1}, \ldots, x_{i}, x_{i+1}, \ldots x_{n}\right)-g^{\sigma}\left(x_{1}, \ldots, x_{i}, x_{i+1}, \ldots x_{n}\right)}{x_{i}-x_{i+1}},
$$

where $g^{\sigma}$ is given by

$$
g^{\sigma}\left(x_{1}, \ldots, x_{i}, x_{i+1}, \ldots x_{n}\right)=g\left(x_{1}, \ldots x_{i-1}, x_{i+1}, x_{i}, x_{i+2} \ldots x_{n}\right) . \quad \text { (see [12]). }
$$

Definition 3 [8] Given an alphabet $E=\left\{e_{1}, e_{2}\right\}$, the symmetrizing operator $\delta_{e_{1} e_{2}}^{k}$ is defined by

$$
\delta_{e_{1} e_{2}}^{k}\left(e_{1}^{n}\right)=\frac{e_{1}^{k+n}-e_{2}^{k+n}}{e_{1}-e_{2}}=S_{k+n-1}\left(e_{1}+e_{2}\right), \quad \text { for all } k, n \in \mathbb{N} .
$$

Definition 4 [9] The symmetrizing operator $\delta_{e_{1} e_{2}}^{-k}$ is defined by

$$
\delta_{e_{1} e_{2}}^{-k} f=\frac{e_{2}^{k} f\left(e_{1}\right)-e_{1}^{k} f\left(e_{2}\right)}{\left(e_{1} e_{2}\right)^{k}\left(e_{1}-e_{2}\right)} \quad(k \in \mathbb{N}) .
$$




\section{Main results}

In this section, we prove the main theorem of the paper which combines all the previously known results in a unified way such that they can be treated as special cases.

Theorem 1 Let $A$ and $E$ be two alphabets, respectively, $\left\{a_{1}, a_{2}\right\}$ and $\left\{e_{1}, e_{2}\right\}$, then we have

$$
\begin{aligned}
\sum_{n=0}^{\infty} S_{n+2}(A) S_{n}(E) z^{n}= & \frac{S_{1}^{2}(A)-S_{2}(-A)-S_{2}(-A) S_{1}(A) S_{1}(E) z}{\prod_{a \in A}\left(1-a e_{1} z\right) \prod_{a \in A}\left(1-a e_{2} z\right)} \\
& +\frac{S_{2}(-E)\left(S_{2}(-A)\right)^{2} z^{2}}{\prod_{a \in A}\left(1-a e_{1} z\right) \prod_{a \in A}\left(1-a e_{2} z\right)}
\end{aligned}
$$

Proof By applying the operator $\delta_{e_{1} e_{2}}^{-1}$ to the series $f\left(e_{1} z\right)=\sum_{n=0}^{\infty} S_{n}(A) e_{1}^{n} z^{n}$, we have

$$
\begin{aligned}
\delta_{e_{1} e_{2}}^{-1} f\left(e_{1} z\right) & =\frac{e_{2} \sum_{n=0}^{\infty} S_{n}(A) e_{1}^{n} z^{n}-e_{1} \sum_{n=0}^{\infty} S_{n}(A) e_{2}^{n} z^{n}}{e_{1} e_{2}\left(e_{1}-e_{2}\right)} \\
& =\frac{\sum_{n=0}^{\infty} S_{n}(A) e_{2} e_{1}^{n} z^{n}-\sum_{n=0}^{\infty} S_{n}(A) e_{1} e_{2}^{n} z^{n}}{e_{1} e_{2}\left(e_{1}-e_{2}\right)} \\
& =\frac{-1}{e_{1} e_{2}}\left(\sum_{n=0}^{\infty} S_{n}(A)\left(\frac{e_{1} e_{2}^{n}-e_{2} e_{1}^{n}}{e_{1}-e_{2}}\right) z^{n}\right) \\
& =\frac{-1}{e_{1} e_{2}}\left(1+\sum_{n=2}^{\infty} S_{n}(A) e_{1} e_{2}\left(\frac{e_{2}^{n-1}-e_{1}^{n-1}}{e_{1}-e_{2}}\right) z^{n}\right) \\
& =\frac{-1}{e_{1} e_{2}}\left(1-e_{1} e_{2} z^{2} \sum_{n=0}^{\infty} S_{n+2}(A) S_{n}(E) z^{n}\right) .
\end{aligned}
$$

On the other hand,

$$
\begin{aligned}
\delta_{e_{1} e_{2}}^{-1} f\left(e_{1} z\right) & =\delta_{e_{1} e_{2}}^{-1}\left(\frac{1}{\prod_{a \in A}\left(1-a e_{1} z\right)}\right) \\
& =\frac{\frac{e_{2}}{\prod_{a \in A}\left(1-a e_{1} z\right)}-\frac{e_{1}}{\prod_{a \in A}\left(1-a e_{2} z\right)}}{e_{1} e_{2}\left(e_{1}-e_{2}\right)} \\
& =\frac{e_{2} \prod_{a \in A}\left(1-a e_{2} z\right)-e_{1} \prod_{a \in A}\left(1-a e_{1} z\right)}{e_{1} e_{2}\left(e_{1}-e_{2}\right) \prod_{a \in A}\left(1-a e_{1} z\right) \prod_{a \in A}\left(1-a e_{2} z\right)} \\
& =\frac{-1}{e_{1} e_{2}}\left(\frac{1-\left(a_{1}+a_{2}\right)\left(e_{1}+e_{2}\right) z+a_{1} a_{2}\left(\left(e_{1}+e_{2}\right)^{2}-e_{1} e_{2}\right) z^{2}}{\prod_{a \in A}\left(1-a e_{1} z\right) \prod_{a \in A}\left(1-a e_{2} z\right)}\right) .
\end{aligned}
$$

So

$$
\begin{aligned}
1-e_{1} e_{2} z^{2} \sum_{n=0}^{\infty} S_{n+2}(A) S_{n}(E) z^{n}= & \frac{1-\left(a_{1}+a_{2}\right)\left(e_{1}+e_{2}\right) z}{\prod_{a \in A}\left(1-a e_{1} z\right) \prod_{a \in A}\left(1-a e_{2} z\right)} \\
& +\frac{a_{1} a_{2}\left(\left(e_{1}+e_{2}\right)^{2}-e_{1} e_{2}\right) z^{2}}{\prod_{a \in A}\left(1-a e_{1} z\right) \prod_{a \in A}\left(1-a e_{2} z\right)} .
\end{aligned}
$$


Therefore

$$
\begin{aligned}
\sum_{n=0}^{\infty} S_{n+2}(A) S_{n}(E) z^{n}= & \frac{S_{1}^{2}(A)-S_{2}(-A)-S_{2}(-A) S_{1}(A) S_{1}(E) z}{\prod_{a \in A}\left(1-a e_{1} z\right) \prod_{a \in A}\left(1-a e_{2} z\right)} \\
& +\frac{S_{2}(-E)\left(S_{2}(-A)\right)^{2} z^{2}}{\prod_{a \in A}\left(1-a e_{1} z\right) \prod_{a \in A}\left(1-a e_{2} z\right)}
\end{aligned}
$$

Thus, this completes the proof.

\section{Generating functions of some well-known numbers}

In this part, we now derive the new generating functions of the products of some known numbers. For the applications of generating functions of some known functions, we refer the reader to see the references [16-29].

For the case $A=\left\{a_{1},-a_{2}\right\}$ and $E=\left\{e_{1},-e_{2}\right\}$ with replacing $a_{2}$ by $-a_{2}, e_{2}$ by $-e_{2}$ in (3.1), we have

$$
\begin{aligned}
\sum_{n=0}^{\infty} S_{n+2}\left(a_{1}+\left[-a_{2}\right]\right) S_{n}\left(e_{1}+\left[-e_{2}\right]\right) z^{n}= & \frac{\left(a_{1}-a_{2}\right)^{2}+a_{1} a_{2}+a_{1} a_{2}\left(e_{1}-e_{2}\right)}{P_{A} P_{E}} \\
& \times \frac{\left(a_{1}-a_{2}\right) z-e_{1} e_{2} a_{1}^{2} a_{2}^{2} z^{2}}{P_{A} P_{E}},
\end{aligned}
$$

with $P_{A} P_{E}=\left(1-a_{1} e_{1} z\right)\left(1+a_{2} e_{1} z\right)\left(1+a_{1} e_{2} z\right)\left(1-a_{2} e_{2} z\right)$.

This case consists of two related parts. Firstly, the substitutions

$$
\left\{\begin{array} { l } 
{ a _ { 1 } - a _ { 2 } = k , } \\
{ a _ { 1 } a _ { 2 } = 1 , }
\end{array} \text { and } \left\{\begin{array}{l}
e_{1}-e_{2}=k, \\
e_{1} e_{2}=1,
\end{array}\right.\right.
$$

in (4.1) give

$$
\sum_{n=0}^{\infty} F_{k, n+2} F_{k, n} z^{n}=\frac{k^{2}+1+k^{2} z-z^{2}}{1-k^{2} z-2\left(k^{2}+1\right) z^{2}-k^{2} z^{3}+z^{4}},
$$

representing a new generating function of $k$-Fibonacci numbers $F_{k, n}$ (with $F_{k, n+2} F_{k, n}=$ $\left.S_{n+2}\left(a_{1}+\left[-a_{2}\right]\right) S_{n}\left(e_{1}+\left[-e_{2}\right]\right)\right)$.

We have the following theorem.

Theorem 2 For $n, k \in \mathbb{N}$, a generating function of the product of $k$-Fibonacci numbers is given by

$$
\sum_{n=0}^{\infty} F_{k, n+1} F_{k, n} z^{n}=\frac{k+k z}{1-k^{2} z-2\left(k^{2}+1\right) z^{2}-k^{2} z^{3}+z^{4}} .
$$

Proof We have

$$
\begin{aligned}
\sum_{n=0}^{\infty} F_{k, n+2} F_{k, n} z^{n} & =\sum_{n=0}^{\infty}\left(k F_{k, n+1}+F_{k, n}\right) F_{k, n} z^{n} \\
& =k \sum_{n=0}^{\infty} F_{k, n+1} F_{k, n} z^{n}+\sum_{n=0}^{\infty} F_{k, n}^{2} z^{n},
\end{aligned}
$$


since

$$
\sum_{n=0}^{\infty} F_{k, n}^{2} z^{n}=\frac{1-z^{2}}{1-k^{2} z-2\left(k^{2}+1\right) z^{2}-k^{2} z^{3}+z^{4}}, \quad(\text { see }[13])
$$

we have

$$
\begin{aligned}
\sum_{n=0}^{\infty} F_{k, n+2} F_{k, n} z^{n}= & \frac{k^{2}+k^{2} z}{1-k^{2} z-2\left(k^{2}+1\right) z^{2}-k^{2} z^{3}+z^{4}} \\
& +\frac{1-z^{2}}{1-k^{2} z-2\left(k^{2}+1\right) z^{2}-k^{2} z^{3}+z^{4}},
\end{aligned}
$$

therefore

$$
\sum_{n=0}^{\infty} F_{k, n+1} F_{k, n} z^{n}=\frac{k+k z}{1-k^{2} z-2\left(k^{2}+1\right) z^{2}-k^{2} z^{3}+z^{4}} .
$$

This completes the proof.

- Based on the relationship (4.2) and (4.3) and with $k=2$, we obtain the following results Corollary 2 For $n \in \mathbb{N}$, a generating function of the product of Pell numbers is given by

$$
\sum_{n=0}^{\infty} P_{n+2} P_{n} z^{n}=\frac{5 z+4 z^{2}-z^{3}}{1-4 z-10 z^{2}-4 z^{3}+z^{4}} .
$$

Corollary 3 For $n \in \mathbb{N}$, a generating function of the product of Pell numbers is given by

$$
\sum_{n=0}^{\infty} P_{n+1} P_{n} z^{n}=\frac{2 z+2 z^{2}}{1-4 z-10 z^{2}-4 z^{3}+z^{4}} .
$$

Secondly, the substitutions

$$
\left\{\begin{array} { l } 
{ a _ { 1 } - a _ { 2 } = 2 } \\
{ a _ { 1 } a _ { 2 } = k }
\end{array} \text { and } \left\{\begin{array}{l}
e_{1}-e_{2}=2 \\
e_{1} e_{2}=k
\end{array},\right.\right.
$$

in (4.1) give

$$
\sum_{n=0}^{\infty} P_{k, n+2} P_{k, n} z^{n}=\frac{(4+k) z+4 k z^{2}-k^{3} z^{3}}{1-4 z-\left(2 k^{2}+8 k\right) z^{2}-4 k^{2} z^{3}+k^{4} z^{4}},
$$

representing a new generating function of the product of $k$-Pell numbers $P_{k, n}$ (with $\left.P_{k, n+2} P_{k, n}=S_{n+1}\left(a_{1}+\left[-a_{2}\right]\right) S_{n-1}\left(e_{1}+\left[-e_{2}\right]\right)\right)$.

We have the following theorem.

Theorem 3 For $n, k \in \mathbb{N}$, a new generating function of the product of $k$-Pell numbers is given by

$$
\sum_{n=0}^{\infty} P_{k, n+1} P_{k, n} z^{n}=\frac{2 z+2 k z^{2}}{1-4 z-\left(2 k^{2}+8 k\right) z^{2}-4 k^{2} z^{3}+k^{4} z^{4}} .
$$

Proof We have

$$
\begin{aligned}
\sum_{n=0}^{\infty} P_{k, n+2} P_{k, n} z^{n} & =\sum_{n=0}^{\infty}\left(2 P_{k, n+1}+k P_{k, n}\right) P_{k, n} z^{n} \\
& =2 \sum_{n=0}^{\infty} P_{k, n+1} P_{k, n} z^{n}+k \sum_{n=0}^{\infty} P_{k, n}^{2} z^{n}
\end{aligned}
$$


since

$$
\sum_{n=0}^{\infty} P_{k, n}^{2} z^{n}=\frac{z-k^{2} z^{3}}{1-4 z-\left(2 k^{2}+8 k\right) z^{2}-4 k^{2} z^{3}+k^{4} z^{4}},
$$

we have

$$
\sum_{n=0}^{\infty} P_{k, n+1} P_{k, n} z^{n}=\frac{1}{2}\left(\sum_{n=0}^{\infty} P_{k, n+2} P_{k, n} z^{n}-k \sum_{n=0}^{\infty} P_{k, n}^{2} z^{n}\right),
$$

therefore

$$
\sum_{n=0}^{\infty} P_{k, n+1} P_{k, n} z^{n}=\frac{2 z+2 k z^{2}}{1-4 z-\left(2 k^{2}+8 k\right) z^{2}-4 k^{2} z^{3}+k^{4} z^{4}} .
$$

This completes the proof.

\section{Generating functions of some well-known polynomials}

In this part, we now derive the new generating functions of the products of some known polynomials. This case consists of two related parts.

Firstly, by making the following restrictions:

$$
\left\{\begin{array} { l } 
{ a _ { 1 } - a _ { 2 } = k , } \\
{ a _ { 1 } a _ { 2 } = 1 , }
\end{array} \text { and } \left\{\begin{array}{l}
e_{1}-e_{2}=x, \\
e_{1} e_{2}=1,
\end{array}\right.\right.
$$

in (4.1), we derive a new generating function, involving the product of $k$-Fibonacci numbers with Fibonacci polynomials

$$
\begin{aligned}
\sum_{n=0}^{\infty} S_{n+2}\left(a_{1}+\left[-a_{2}\right]\right) S_{n}\left(e_{1}+\left[-e_{2}\right]\right) z^{n} & =\frac{k^{2}+1+k x z-z^{2}}{1-k x z-\left(k^{2}+x^{2}+2\right) z^{2}-k x z^{3}+z^{4}} \\
& =\sum_{n=0}^{\infty} F_{k, n+2} F_{n}(x) z^{n} .
\end{aligned}
$$

Corollary 4 For $n, k \in \mathbb{N}$, we have

$$
F_{k, n+2} F_{n}(x)=S_{n+2}\left(a_{1}+\left[-a_{2}\right]\right) S_{n}\left(e_{1}+\left[-e_{2}\right]\right) .
$$

We deduce the following theorem.

Theorem 4 For $n \in \mathbb{N}$, a new generating function of the product of $k$-Fibonacci numbers with Fibonacci polynomials is given by

$$
\sum_{n=0}^{\infty} F_{k, n+1} F_{n}(x) z^{n}=\frac{k+x z}{1-k x z-\left(k^{2}+x^{2}+2\right) z^{2}-k x z^{3}+z^{4}} .
$$

Proof We have

$$
\begin{aligned}
\sum_{n=0}^{\infty} F_{k, n+2} F_{n}(x) z^{n} & =\sum_{n=0}^{\infty}\left(k F_{k, n+1}+F_{k, n}\right) F_{n}(x) z^{n} \\
& =k \sum_{n=0}^{\infty} F_{k, n+1} F_{n}(x) z^{n}+\sum_{n=0}^{\infty} F_{k, n} F_{n}(x) z^{n}
\end{aligned}
$$


Since

$$
\sum_{n=0}^{\infty} F_{k, n} F_{n}(x) z^{n}=\frac{1-z^{2}}{1-k x z-\left(k^{2}+x^{2}+2\right) z^{2}-k x z^{3}+z^{4}}(\text { see [14]) }
$$

we have

$$
\begin{aligned}
\sum_{n=0}^{\infty} F_{k, n+2} F_{n}(x) z^{n}= & \frac{k^{2}+k x z}{1-k x z-\left(k^{2}+x^{2}+2\right) z^{2}-k x z^{3}+z^{4}} \\
& +\frac{1-z^{2}}{1-k x z-\left(k^{2}+x^{2}+2\right) z^{2}-k x z^{3}+z^{4}}
\end{aligned}
$$

therefore

$$
\sum_{n=0}^{\infty} F_{k, n+1} F_{n}(x) z^{n}=\frac{k+x z}{1-k x z-\left(k^{2}+x^{2}+2\right) z^{2}-k x z^{3}+z^{4}} .
$$

This completes the proof.

- Based on the relationship (5.1) and (5.2) and with $k=2$, we obtain the following results.

Corollary 5 For $n \in \mathbb{N}$, a new generating function of the product of Pell numbers with Fibonacci polynomials is given by

$$
\sum_{j=0}^{\infty} P_{n+2} F_{n-1}(x) z^{n}=\frac{5 z+2 x z^{2}-z^{3}}{1-2 x z-\left(x^{2}+6\right) z^{2}-2 x z^{3}+z^{4}} .
$$

Corollary 6 For $n \in \mathbb{N}$, a new generating function of the product of Pell numbers with Fibonacci polynomials is given by

$$
\sum_{n=0}^{\infty} P_{n+1} F_{n-1}(x) z^{n}=\frac{2 z+x z^{2}}{1-2 x z-\left(x^{2}+6\right) z^{2}-2 x z^{3}+z^{4}} .
$$

Secondly, the substitutions

$$
\left\{\begin{array} { l } 
{ a _ { 1 } - a _ { 2 } = 2 , } \\
{ a _ { 1 } a _ { 2 } = k , }
\end{array} \text { and } \left\{\begin{array}{l}
e_{1}-e_{2}=x, \\
e_{1} e_{2}=1,
\end{array}\right.\right.
$$

in (4.1) give

$$
\sum_{n=0}^{\infty} P_{k, n+2} F_{n-1}(x) z^{n}=\frac{(4+k) z+2 k x z^{2}-k^{2} z^{3}}{1-2 x z-\left(k\left(x^{2}+2\right)+4\right) z^{2}-2 k x z^{3}+k^{2} z^{4}},
$$

representing a new generating function of the product of $k$-Pell numbers with Fibonacci polynomials, and also we have

$$
P_{k, n+2} F_{n-1}(x)=S_{n+1}\left(a_{1}+\left[-a_{2}\right]\right) S_{n-1}\left(e_{1}+\left[-e_{2}\right]\right) .
$$

- For the case $A=\left\{a_{1},-a_{2}\right\}$ and $E=\left\{2 e_{1},-2 e_{2}\right\}$ with replacing $a_{2}$ by $-a_{2}, e_{1}$ by $2 e_{1}$ and $e_{2}$ by $-2 e_{2}$ in (3.1), we have

$$
\begin{aligned}
& \sum_{n=0}^{\infty} S_{n+2}\left(a_{1}+\left[-a_{2}\right]\right) S_{n}\left(2 e_{1}+\left[-2 e_{2}\right]\right) z^{n}=\frac{\left(a_{1}-a_{2}\right)^{2}+a_{1} a_{2}}{P_{A} P_{E^{*}}} \\
& \quad+\frac{2 a_{1} a_{2}\left(a_{1}-a_{2}\right)\left(e_{1}-e_{2}\right) z}{P_{A} P_{E^{*}}}-\frac{4 e_{1} e_{2}\left(a_{1} a_{2}\right)^{2} z^{2}}{P_{A} P_{E^{*}}}
\end{aligned}
$$

with $P_{A} P_{E^{*}}=\left(1-2 a_{1} e_{1} z\right)\left(1+2 a_{1} e_{2} z\right)\left(1+2 a_{2} e_{1} z\right)\left(1-2 a_{2} e_{2} z\right)$. 
This case consists of tow related parts. Firstly, the substitutions

$$
\left\{\begin{array} { l } 
{ a _ { 1 } - a _ { 2 } = k , } \\
{ a _ { 1 } a _ { 2 } = 1 , }
\end{array} \text { and } \left\{\begin{array}{l}
e_{1}-e_{2}=x \\
4 e_{1} e_{2}=-1
\end{array}\right.\right.
$$

in (5.3) give

$$
\begin{aligned}
\sum_{n=0}^{\infty} S_{n+2}\left(a_{1}+\left[-a_{2}\right]\right) S_{n}\left(2 e_{1}+\left[-2 e_{2}\right]\right) z^{n} & =\frac{k^{2}+1+2 k x z+z^{2}}{1-2 k x z-\left(4 x^{2}-k^{2}-2\right) z^{2}+2 k x z^{3}+z^{4}} \\
& =\sum_{n=0}^{\infty} F_{k, n+2} U_{n}(x) z^{n}
\end{aligned}
$$

representing a new generating function of the product of $k$-Fibonacci numbers with Tchebychev polynomials of second kind.

Corollary 7 For $n, k \in \mathbb{N}$, we have

$$
F_{k, n+2} U_{n}(x)=S_{n+2}\left(a_{1}+\left[-a_{2}\right]\right) S_{n}\left(2 e_{1}+\left[-2 e_{2}\right]\right) .
$$

We deduce the following theorem.

Theorem 5 We have the following a new generating function of the product of $k$-Fibonacci numbers and Tchebychev polynomials of second kind as

$$
\sum_{n=0}^{\infty} F_{k, n+1} U_{n}(x) z^{n}=\frac{k+2 x z}{1-2 k x z-\left(4 x^{2}-k^{2}-2\right) z^{2}+2 k x z^{3}+z^{4}} .
$$

Proof We have

$$
\begin{aligned}
\sum_{n=0}^{\infty} F_{k, n+2} U_{n}(x) z^{n} & =\sum_{n=0}^{\infty}\left(k F_{k, n+1}+F_{k, n}\right) U_{n}(x) z^{n} \\
& =k \sum_{n=0}^{\infty} F_{k, n+1} U_{n}(x) z^{n}+\sum_{n=0}^{\infty} F_{k, n} U_{n}(x) z^{n}
\end{aligned}
$$

since

$$
\sum_{n=0}^{\infty} F_{k, n} U_{n}(x) z^{n}=\frac{1+z^{2}}{1-2 k x z-\left(4 x^{2}-k^{2}-2\right) z^{2}+2 k x z^{3}+z^{4}}, \quad \text { (see [13]) }
$$

we have

$$
\begin{aligned}
\sum_{n=0}^{\infty} F_{k, n+2} U_{k, n} z^{n}= & \frac{k^{2}+2 k x z}{1-2 k x z-\left(4 x^{2}-k^{2}-2\right) z^{2}+2 k x z^{3}+z^{4}} \\
& +\frac{1+z^{2}}{1-2 k x z-\left(4 x^{2}-k^{2}-2\right) z^{2}+2 k x z^{3}+z^{4}},
\end{aligned}
$$

therefore

$$
\sum_{n=0}^{\infty} F_{k, n+1} U_{n}(x) z^{n}=\frac{k+2 x z}{1-2 k x z-\left(4 x^{2}-k^{2}-2\right) z^{2}+2 k x z^{3}+z^{4}} .
$$

This completes the proof. 
- Based on the relationship (5.4) and (5.5) and with $k=2$, we obtain the following results.

Corollary 8 For $n \in \mathbb{N}$, a new generating function of the product of Pell numbers and Tchebychev polynomials of second kind is given by

$$
\sum_{n=0}^{\infty} P_{n+2} U_{n-1}(x) z^{n}=\frac{5 z+4 x z^{2}+z^{3}}{1-4 x z-\left(4 x^{2}-6\right) z^{2}+4 x z^{3}+z^{4}} .
$$

Corollary 9 For $n \in \mathbb{N}$, a new generating function of the product of Pell numbers and Tchebychev polynomials of second kind is given by

$$
\sum_{n=0}^{\infty} P_{n+1} U_{n-1}(x) z^{n}=\frac{2 z+2 x z^{2}}{1-4 x z-\left(4 x^{2}-6\right) z^{2}+4 x z^{3}+z^{4}} .
$$

Secondly, the substitutions the substitutions

$$
\left\{\begin{array} { l } 
{ a _ { 1 } - a _ { 2 } = 2 , } \\
{ a _ { 1 } a _ { 2 } = k , }
\end{array} \text { and } \left\{\begin{array}{l}
e_{1}-e_{2}=x, \\
4 e_{1} e_{2}=-1,
\end{array}\right.\right.
$$

in (5.3) give

$$
\sum_{n=0}^{\infty} P_{k, n+2} U_{n-1}(x) z^{n}=\frac{(4+k) z+4 k x z^{2}+k^{2} z^{3}}{1-4 x z-\left(4 k x^{2}-2 k-4\right) z^{2}+4 k x z^{3}+k^{2} z^{4}},
$$

representing a new generating function of the product of $k$-Pell numbers with Tchebychev polynomials of second kind, and also we have

$$
P_{k, n+2} U_{n-1}(x)=S_{n+1}\left(a_{1}+\left[-a_{2}\right]\right) S_{n-1}\left(2 e_{1}+\left[-2 e_{2}\right]\right) .
$$

Theorem 6 For $n \in \mathbb{N}$, a new generating function of the product of Fibonacci numbers $F_{n}$ and Tchebychev polynomials of first kind is given by

$$
\sum_{n=0}^{\infty} F_{n+2} T_{n}(x) z^{n}=\frac{2-x z+\left(1-4 x^{2}\right) z^{2}-x z^{3}}{1-2 x z-\left(4 x^{2}-3\right) z^{2}+2 x z^{3}+z^{4}} .
$$

Proof We see that

$$
\begin{aligned}
\sum_{n=0}^{\infty} F_{n+2} T_{n}(x) z^{n} & \\
= & \sum_{n=0}^{\infty} S_{n+2}\left(a_{1}+\left[-a_{2}\right]\right)\left(S_{n}\left(2 e_{1}+\left[-2 e_{2}\right]\right)-x S_{n-1}\left(2 e_{1}+\left[-2 e_{2}\right]\right)\right) z^{n} \\
= & \sum_{n=0}^{\infty} S_{n+2}\left(a_{1}+\left[-a_{2}\right]\right) S_{n}\left(2 e_{1}+\left[-2 e_{2}\right]\right) z^{n} \\
& -x \sum_{n=0}^{\infty} S_{n+2}\left(a_{1}+\left[-a_{2}\right]\right) S_{n-1}\left(2 e_{1}+\left[-2 e_{2}\right]\right) z^{n} \\
= & \sum_{n=0}^{\infty} F_{n+2} U_{n}(x) z^{n}-\frac{x}{2\left(e_{1}+e_{2}\right)} \sum_{n=0}^{\infty} S_{n+2}\left(a_{1}+\left[-a_{2}\right]\right)\left(\left(2 e_{1}\right)^{n}-\left(-2 e_{2}\right)^{n}\right) z^{n} \\
= & \sum_{n=0}^{\infty} F_{n+2} U_{n}(x) z^{n}-\frac{x}{2\left(e_{1}+e_{2}\right)}\left(\begin{array}{c}
\sum_{n=0}^{\infty} S_{n+2}\left(a_{1}+\left[-a_{2}\right]\right)\left(2 e_{1} z\right)^{n} \\
\left.-\sum_{n=0}^{\infty} S_{n+2}\left(a_{1}+\left[-a_{2}\right]\right)\left(-2 e_{2} z\right)^{n}\right) .
\end{array}\right.
\end{aligned}
$$


On the other hand, we know that

$$
\sum_{n=0}^{\infty} S_{n+2}\left(a_{1}+\left[-a_{2}\right]\right) z^{n}=\frac{2+z}{1-z-z^{2}}, \quad(\text { see }[8])
$$

from which it follows

$$
\begin{aligned}
\sum_{n=0}^{\infty} F_{n+2}\left(a_{1}+\left[-a_{2}\right]\right) T_{n}(x) z^{n}= & \frac{2+2 x z+z^{2}}{1-2 x z-\left(4 x^{2}-3\right) z^{2}+2 x z^{3}+z^{4}}-\frac{x}{2\left(e_{1}+e_{2}\right)} \\
& \times\left(\frac{2+2 e_{1} z}{1-2 e_{1} z-4 e_{1}^{2} z^{2}}-\frac{2-2 e_{2} z}{1+2 e_{2} z-4 e_{2}^{2} z^{2}}\right),
\end{aligned}
$$

therefore

$$
\sum_{n=0}^{\infty} F_{n+2}\left(a_{1}+\left[-a_{2}\right]\right) T_{n}(x) z^{n}=\frac{2-x z+\left(1-4 x^{2}\right) z^{2}-x z^{3}}{1-2 x z-\left(4 x^{2}-3\right) z^{2}+2 x z^{3}+z^{4}} .
$$

This completes the proof.

Theorem 7 A new generating function of the product of Fibonacci numbers $F_{n}$ and Tchebychev polynomials of first kind is given by

$$
\sum_{n=0}^{\infty} F_{n+1} T_{n}(x) z^{n}=\frac{1-2 x^{2} z^{2}-x z^{3}}{1-2 x z-\left(4 x^{2}-3\right) z^{2}+2 x z^{3}+z^{4}} .
$$

Proof We have that

$$
\begin{aligned}
\sum_{n=0}^{\infty} F_{n+2} T_{n}(x) z^{n} & =\sum_{n=0}^{\infty}\left(F_{n+1}+F_{n}\right) T_{n}(x) z^{n} \\
& =\sum_{n=0}^{\infty} F_{n+1} T_{n}(x) z^{n}+\sum_{n=0}^{\infty} F_{n} T_{n}(x) z^{n} .
\end{aligned}
$$

Since

$$
\sum_{n=0}^{\infty} F_{n} T_{n}(x) z^{n}=\frac{1-x z-\left(1-2 x^{2}\right) z^{2}}{1-2 x z-\left(4 x^{2}-3\right) z^{2}+2 x z^{3}+z^{4}},(\text { see }[15])
$$

therefore

$$
\sum_{n=0}^{\infty} F_{n+1} T_{n}(x) z^{n}=\frac{1-2 x^{2} z^{2}-x z^{3}}{1-2 x z-\left(4 x^{2}-3\right) z^{2}+2 x z^{3}+z^{4}} .
$$

This completes the proof.

\section{Conclusion}

In this paper, we have derived new theorems in order to determine generating functions of $k$-Fibonacci and $k$-Pell numbers and Fibonacci polynomails and Tchebychev polynomials of the first and second kinds. The derived theorems and corollaries are based on symmetric functions and products of these numbers and polynomials. 


\section{References}

1. Pintér, A., Srivastava, H.M.: Generating functions of the incomplete Fibonacci and Lucas numbers. Rend. Circ. Mat. Palermo 48, 591-596 (1999)

2. Djordjevic, G.B.: Generating functions of the incomplete generalized Fibonacci and generalized Lucas numbers. Fibonacci Q. 42, 106-113 (2004)

3. Djordjevic, G.B., Srivastava, H.M.: Incomplete generalized Jacobsthal and Jacobsthal-Lucas numbers. Math. Comput. Model. 42, 1049-1056 (2005)

4. Tasci, D., Cetin Firengiz, M.: Incomplete Fibonacci and Lucas p-numbers. Math. Comput. Model. 52, 1763-1770 (2010)

5. Falcon, S., Plaza, A.: On the Fibonacci $k$-numbers. Chaos Sulut. Fract. 32, 1615-1624 (2007)

6. Catarino, P.: On some identities and generating functions for $k$-Pell numbers. Int. J. Math. Anal. 7, 1877-1884 (2013)

7. Boussayoud, A.: On some identities and generating functions for Pell-Lucas numbers. Online J. Anal. Comb. 12, 1-10 (2017)

8. Boussayoud, A., kerada, M., Boulyer, M.: A simple and accurate method for determination of some generalized sequence of numbers. Int. J. Pure Appl. Math. 108, 503-511 (2016)

9. Boussayoud, A., Sahali, R.: The application of the operator $L_{b_{1} b_{2}}^{-k}$ in the series $\sum_{j=0}^{+\infty} a_{j} b_{1}^{j} z^{j}$. J. Adv. Res. Appl. Math. 7, 68-75 (2015)

10. Abderrezzak, A.: Généralisation de la transformation d'Euler d'une série formelle. Adv. Math. 103, 180-195 (1994)

11. Abderrezzak, A.: Généralisation d'identité s de Carlitz. Howard et Lehmer. Aequ. Math. 49, 36-46 (1995)

12. Macdonald, I.G.: Symmetric Functions and Hall Polynomias. Oxford University Press, Oxford (1979)

13. Boussayoud, A., Harrouche, N.: Complete symmetric functions and $k$-Fibonacci numbers. Commun. Appl. Anal. 20, 457-465 (2016)

14. Boussayoud, A., Kerada, M., Araci, S.: Symmetric functions of the $k$-Fibonacci and $k$-Lucas numbers. Int. J. Adv. Appl. Sci. (accepted)

15. Foata, D., Han, G-N.: Nombres de Fibonacci et Polynômes Orthog onaux. In: Leonardo Fibonacci : il tempo, le opere, l'eredità scientifica, pp. 179-200 (1994)

16. Boussayoud, A., Kerada, M., Harrouche, N.: On the $k$-Lucas numbers and Lucas Polynomials. Turk. J. Anal. Numb. 5, 121-125 (2017)

17. Boussayoud, A., Kerada, M., Sahali, R.: Symmetrizing operations on some orthogonal polynomails. Int. Electron. J. Pure Appl. Math. 9, 191-199 (2015)

18. Boussayoud, A., Kerada, M.: Symmetric and generating functions. Int. Electron. J. Pure Appl. Math. 7, 195-203 (2014)

19. Boussayoud, A., Kerada, M., Sahali, R., Rouibah, W.: Some applications on generating functions. J. Concr. Appl. Math. 12, 321-330 (2014)

20. Horadam, A.F., Mahon, J.M.: Pell and Pell-Lucas polynomials. Fibonacci Q. 23, 7-20 (1985)

21. Horadam, A.F.: Generating functions for powers of a certain generalized sequence of numbers. Duke Math. J. 32, 437-446 (1965)

22. Bolat, C., Kose, H.: On the properties of $k$-Fibonacci numbers. Int. J. Contemp. Math. Sci. 5, 1097-1105 (2010)

23. Kruchinin, D.V., Kruchinin, V.V.: Application of a composition of generating functions for obtaining explicit formulas of polynomials. J. Math. Anal. Appl. 404, 161-171 (2013)

24. Mezo, I.: Several generating functions for second-order recurrence sequences. J. Integer Seq. 12, 1-16 (2009)

25. Kilic, E.: Sums of the squares of terms of the sequence $\left\{U_{n}\right\}$. Proc. Indian Acad. Sci. Math. Sci. 118, 27-41 (2008)

26. Araci, S.: Novel identities involving Genocchi numbers and polynomials arising from applications of umbral calculus. Appl. Math. Comput. 233, 599-607 (2014)

27. Falcon, S.: On the sequences of products of two $k$-Fibonacci numbers. Am. Rev. Math. Stat. 2, 111-120 (2014)

28. Falcon, S., Plaza, A.: On $k$-Fibonacci sequences and polynomials and their derivatives. Chaos Sulut. Fract. 39, 1005-1019 (2009)

29. Falcon, S., Plaza, A.: The $k$ - Fibonacci sequence and the Pascal 2-triangle. Chaos Sulut. Fract. 33, 38-49 (2008)

Publisher's Note Springer Nature remains neutral with regard to jurisdictional claims in published maps and institutional affiliations. 